\title{
Hemoglobin A1c measurement for the diagnosis of Type 2 diabetes in children
}

\author{
Chirag Kapadia1*, Philip Zeitler ${ }^{2}$ and Drugs and Therapeutics Committee of the Pediatric Endocrine Society
}

\begin{abstract}
Laboratory measurements of hemoglobin A1c above $6.5 \%$ were approved as an additional diagnostic criteria for diabetes mellitus by the American Diabetes Association in 2010. Several recent pediatric studies have cast HbA1c measurement in children in an unfavorable light in the pediatric population, by comparing $\mathrm{HbA1c}$ measurements to results on oral glucose tolerance test (OGT) or fasting plasma glucose (FPG). However, many of these studies do not recognize that diabetes diagnostic criteria are based upon long-term health outcomes. In this sense, OGTT and FPG have themselves never been validated in the pediatric population. Studies to validate diagnostic tests for diabetes in pediatric populations may take a substantial period of time, and may prove unfeasible. However, studies that tie diagnostic results as a child to diagnostic results as an adult may be more feasible and may provide the data needed to determine which pediatric diagnostic criteria to use. Thus, for the time being, except for cases of hemoglobinopathy, cystic fibrosis, and a few other exceptions, describing HbA1c as 'lacking in sensitivity or specificity' in the pediatric population because of lack of correlation with OGTT is not scientifically sound.
\end{abstract}

Keywords: Hemoglobin A1c, Type 2 diabetes, Pediatrics, OGTT, Retinopathy, Diagnosis

\section{Background}

This paper is a commentary from the Pediatric Endocrine Society Drugs and Therapeutics Committee. Its goal is provide background information and guidance to both pediatric endocrinologists, and also to primary care practictioners on the current use of HbA1c for the diagnosis of Type 2 diabetes in children. In addition, it has the goal of pointing out some of the flaws in current methodologies being used to address this question.

Diagnostic criteria for diabetes were initially was based on variation from normal [1]. As information about the degrees of glycemia that lead to diabetic complications became available, criteria were revised. In 1997, the Expert Committee on Diagnosis and Classification of Diabetes Mellitus examined population data for retinopathy, and noted that for Fasting Plama Glucose (FPG), 2-hour postload glucose during an oral glucose tolerance test (OGTT), and hemoglobin $\mathrm{A}_{1 \mathrm{c}}(\mathrm{HbA1c})$, the diabetes-related complication of retinopathy increased linearly above a certain cutpoints; for FPG and OGTT,

\footnotetext{
* Correspondence: ckapadia@phoenixchildrens.com

'Phoenix Children's Hospital, Phoenix, AZ 85016, USA

Full list of author information is available at the end of the article
}

those cutpoints became the basis for the diagnosis of diabetes [2].

In 2010, it was felt that with the increasing adherence to the National Glycohemoglobin Standardization Program (NGSP), laboratory-based HbA1c is measured in a standardized fashion in the majority of labs in the U.S. Furthermore, the American Diabetes Association (ADA) noted that review of epidemiologic data supported a relationship between $\mathrm{HbA1c}$ and the risk of retinopathy similar to what had been shown for FPG and OGTT. Thus, in 2010, the ADA added A1c of 6.5 percent or greater as a diagnostic criterion [3].

The ADA required that a laboratory-based HbA1c assay method certified by the NGSP be used. This ensures that the assay used is standardized or traceable to the Diabetes Control And Complications Trial $[4,5]$.

Obtaining screening laboratories for diabetes in the atrisk population has long been established practice in adults. However, in the pediatric population, data have thus far largely been extrapolated from adult studies, and screening practices vary. The issue has become more pertinent with the rise of pediatric obesity. While FPG and OGTT thresholds, as extrapolated from adult populations, have largely been accepted by the community
Ciomed Central

(c) 2012 Kapadia and Zeitler; licensee BioMed Central Ltd. This is an Open Access article distributed under the terms of the Creative Commons Attribution License (http://creativecommons.org/licenses/by/2.0), which permits unrestricted use, distribution, and reproduction in any medium, provided the original work is properly cited. 
of pediatric practictioners, the more recent recommendation of use of HbA1c has met resistance.

\section{Main text and discussion}

An important benefit of use of $\mathrm{HbA1c}$ is that patient does not need to be fasting, and testing does not require a return visit [6]. HbA1c has less variability and is more reproducible than FPG and OGTT [7].

However, HbA1c also has several potential disadvantages. Hb A1c may miss cases of Type 1 diabetes in which hyperglycemia develops over a short period of time. Furthermore, HbA1c is not a perfect estimation of mean blood glucose, and varies by ethnicity $[8,9]$. In addition, diseases such as iron deficiency anemia, sicklecell disease, thalassemia, and other hemoglobinopathies, can alter HbA1c [10].

\section{Recent studies}

When FPG was used to diagnose diabetes, HbA1c of $6.5 \%$ had sensitivity of $75.0 \%$ and specificity of $99.9 \%$ [11]. The authors examined the diagnosis of pre-diabetes noted a low sensitivity but a good specificity (98.3\%) for a HbA1c of $5.7 \%$, and also for HbA1c of $6.0 \%$ (specificity 99.4\%).

Another study [12] compared HbA1c to OGTT in over 1000 obese patients and concluded that A1c has low sensitivity and specificity for diabetes when diabetes is defined by OGTT results; 9 of 893 patients with an HbA1c less than 5.7\% were determined to have diabetes using OGTT criteria. In addition, a larger number of cases of prediabetes defined by OGTT were not identified using an HbA1c cut-off of 5.7\%; 240 of the 347 (69\%) cases of prediabetes in this high-risk population had HbA1c < $5.7 \%$.

In a smaller study, HBA1c cut-off of $6.5 \%$ had a sensitivity of $40 \%$ and a specificity of $96 \%$ in accurately diagnosing patients with type 2 diabetes, when using OGTT as a gold standard [13].

A study of 254 overweight or obese adolescents also raised questions about HbA1c use for diagnosis [14]. In this study, there were 99 (39\%) cases of prediabetes and $3(1.2 \%)$ cases of diabetes using FPG and OGTT as gold standards. Test performance was assessed using receiver operating characteristic (ROC) curves and calculations of area under the ROC curve (AUC). HbA1c (AUC 0.54 [95\% CI 0.47-0.61]) displayed poor discrimination for identifying children with dysglycemia that had been identified on OGTT. In fact, in this study, random glucose (AUC 0.66 [0.60-0.73]) had better correlation with OGTT.

The potential advantages of use of $\mathrm{HbA1c}$, however, were born out by a study before and after a change in recommendation to allow use of $\mathrm{HbA1c}$ in screening of adolescents [15]. Rates of screening for diabetes increased from 39 to $47 \%$ as a result, and this led to twice as many incident T2DM diagnoses during a similar time period. HbA1c threshold of $6 \%$ indicated progression to diabetes in $18 \%$ of patients, while the $5.7 \%$ threshold resulted in only $1.3 \%$ progression to diabetes, over about 3 years. In a separate study with 468 subjects, HbA1c cut-off of $6 \%$ had greater correlation with OGTT results than A1c threshold of 5.7\% [16]. This study showed sensitivity and specificity of $86 \%$ and $85 \%$, respectively, for $\mathrm{HbA1c}$ threshold $5.7 \%$, but $99 \%$ and $96 \%$, respectively, for $\mathrm{HbaA} 1 \mathrm{c}$ threshold of $6 \%$.

\section{Comparing unvalidated methodologies: a pitfall of many studies}

Studies that compare HbA1c to other methods of diagnosing diabetes in pediatric populations are handicapped by the fact that the other methods - FPG and OGTT are themselves not validated in the pediatric population. A truly validated definition of diabetes in pediatric populations requires insight into the relationship of the proposed definitions to relevant aspects of medium and long-term health [17].

Assuming that OGTT or FPG are better than HbA1c for determining risk of complications is unfounded; in adults, even though HbA1c, FPG, and OGTT are often discrepant in individuals, all 3 markers are shown to correlate very well with risk of complications [2,3].

\section{Conclusions}

The diagnostic thresholds of glycemia in the adult population were formulated because these are theh thresholds at which retinopathy increases. Such thresholds have never been defined in the pediatric population. In this sense, for diagnosis of diabetes in asymptomatic or minimally symptomatic children, there are no validated methodologies. OGTT, which is being used as a 'gold standard' in many studies, in addition to lacking validation as noted above, also suffers from having low reproducibility [18]. Therefore, dismissal of HbA1c $6.5 \%$ or greater for diagnosis of diabetes at this time, because of lack of correlation with OGTT, is a flawed approach.

The lack of correlation between studies has already been acknowledged and discussed in adult populations, including in the ADA's clinical practice guidelines $[3,4]$. While the correlation appears to be lower in pediatrics, it is not yet clear which of the studies, if any, are 'faulty', and obtaining such data would require comprehensive, multi-center, long-term studies on incidence of retinopathy; such studies appear unlikely to occur at this time.

Therefore, conclusions that dismiss HbA1c use for the diagnosis of diabetes in children are based on incomplete data. Considering that the demographics of Type 2 diabetes skew towards disadvantated populations, we should not dismiss a valuable, flexible tool that, put into 
widespread use, may in fact increase, not decrease, early detection of this disease [15].

\section{Recommendations}

1. ADA criteria for the diagnosis of diabetes, though formulated from data in adults, are useful for screening the asymptomatic, or minimally symptomatic, at-risk pediatric population at this time, and this includes the ADA's more recent recommendations regarding $\mathrm{HbA1c}$.

2. When test results do not correlate with each other, or appear to give information conflicting to the clinical situation, practictioners must use clinical judgement. Amongst FPG, OGTT, or HbA1c, one test is not validated to a greater extent than another in the pediatric population.

3. There are some questions about HbA1c use in those with cystic fibrosis [19], though some data shows it may be useful [20].

4. HbA1c is likely reliable in those with sickle-cell carrier status, as long as an assay without interference from abnormal hemoglobins is used $[5,21]$. HbA1c is not reliable in those known to have a hemoglobinopathy or any other disorder resulting in significant increases in red blood cell turnover, or in pregnancy [22]. Assay interference information is available on the NGSP website [5].

5. Laboratory-based hemoglobin A1c using a methodology and assay certified by the National Glycohemoglobin Standardization Program is the preferred method for diagnostic purposes [3-5].

\section{Abbreviations}

ADA: American Diabetes Association; FPG: Fasting Plasma Glucose;

OGTT: Oral Glucose Tolerance Test; HbA1c: Hemoglobin A1c; NGSP: National Glycohemoglobin Standardization Program.

\section{Competing interests}

There are no competing interest for any participating authors or collaborators.

\section{Authors' contributions}

$\mathrm{CK}$ and $\mathrm{PZ}$ are the primary authors and editors of this commentary. The remaining collaborators from the PES Drugs and Therapeutics Committee helped shape the article through discussion at meetings and conference calls, and provided editing of the manuscript. All authors read and approved the final manuscript.

\section{Authors' information}

Pediatric Endocrine Society Drugs and Therapeutics Committee for 2011-2012: Kapadia C $C^{\mathrm{a}}$, Zeitler P $\mathrm{P}^{\mathrm{b}}$, Divall, $\mathrm{S}^{\mathrm{c}}$, Grimberg, Ad, Gitelman SE', Quintos JB', Draznin $M^{9}$, Nebesio $T^{h}$, Myers SE', Potter $A^{j}$, Vogiatzi $M^{k}$, Raman $S^{\prime}$, Waguespack $\mathrm{SG}^{\mathrm{m}}$, Eugster $\mathrm{E}^{\mathrm{h}}$, Boney $\mathrm{CM}^{\mathrm{f}}$, Rosenthal $\mathrm{SM}^{\mathrm{e}}$, Silverstein $\mathrm{J}^{\mathrm{n}}$ a. Phoenix Children's Hospital, Phoenix, AZ b. Department of Pediatrics, University of Colorado, Denver, CO c. Johns Hopkins University, Baltimore, MD

d. Children's Hospital of Philadelphia, Philadelphia, PA

e. University of California-San Francisco, San Francisco, CA

f. Rhode Island Hospital, The Warren Alpert Medical School of Brown

University, Providence, Rl g. Michigan State University Kalamazoo Center for Medical Studies, Kalamazoo, MI

h. Riley Children's Hospital, Indianapolis, IN

i. Cardinal Glennon Children's Hospital, St Louis, MO

j. Vanderbilt University Medical Center, Nashville, TN

k. Weill Medical Center of Cornell University, NY, NY

I. Children's Mercy Hospital, Kansas City, KS

m. University of Texas M.D. Anderson Cancer Center, Houston, TX

n. University of Florida College of Medicine, Gainesville, FL

\section{Author details}

'Phoenix Children's Hospital, Phoenix, AZ 85016, USA. ²Department of Pediatrics, University of Colorado, Denver, CO, USA.

Received: 7 November 2012 Accepted: 12 November 2012 Published: 20 December 2012

\section{References}

1. National Diabetes Data Group: Classification and diagnosis of diabetes mellitus and other categories of glucose intolerance. Diabetes 1979, 28:1039-1057.

2. Expert Committee on the Diagnosis and Classification of Diabetes Mellitus: Report of the expert committee on the diagnosis and classification of diabetes mellitus. Diabetes Care 1997, 20:1183-1197.

3. International Expert Committee: International Expert Committee report on the role of the A1C assay in the diagnosis of diabetes. Diabetes Care 2009, 32:1327-1334.

4. American Diabetes Association: Standards of medical care in diabetes -2010. Diabetes Care 2010, 33:511-561.

5. National Glycohemoglobin Standardization Program: www.ngsp.org.

6. Ehehalt S, Gauger N, Blumenstock G, Feldhahn L, Scheffner T, Schweizer R, et al: Hemoglobin A1c is a reliable criterion for diagnosing Type 1 diabetes in childhood and adolescence. Pediatric Diabetes 2010, doi:10.1111/j.1399-5448.2009.00633.x.

7. Lippi G, Targer G: A laboratory standpoint on the role of hemoglobin A1c for the diagnosis of diabetes in childhood: more doubts than certainties? Pediatric Diabetes 2010, doi:10.1111/j.1399-5488.

8. Diabetes Research in Children Network (DirecNet) Study Group, Wilson DM, Kollman C: Relationship of A1c to glucose concentrations in children with Type 1 diabetes: assessments by high-frequency glucose determinations by sensors. Diabetes Care 2008, 31:381-385.

9. Kamps JL, Hempe JM, Chalew SA: Racial Disparity in hemoglobin A1c in independent of mean blood glucose in children with Type 1 diabetes. Diabetes Care 2010, 33:951-957.

10. Tarim O, Kucukerdogan A, Gunay U, Eralp O, Ercan I: Effects of iron deficiency anemia on hemoglobin A1c in Type 1 diabetes mellitus. Pediatr Int 1999, 41:357-362.

11. Lee JM, Wu E, Tarini B, Herman WH, Yoon E: Diagnosis of diabetes using hemoglobin A1c: should recommendations in adults Be extrapolated to adolescents? J Pediatr 2011, 158(6):947-952.

12. Nowicka P, Santoro N, Liu H, Lartaud D, Shaw MM, Goldberg R, et al: Utility of Hemoglobin A1c for diagnosing diabetes and prediabetes in obese children and adolescents. Diabetes Care 2011, 34:1306-1311.

13. Babar GS, Sherman AK: Comparison of HbA1C and OGTT for the diagnosis of type-2 diabetes mellitus in overweight children and adolescents, Abstract presented at pediatric academic societies meeting. Colorado, United States: Denver; 2011.

14. Lee JM, Gebremariam A, Wu EL, LaRose J, Gurney JG: Evaluation of nonfasting tests to screen for childhood and adolescent dysglycemia. Diabetes Care 2011, 34(12):2597-2602.

15. Love-Osborne K, Sheeder J, Svircev A, et al HbA1c: Increasing Diabetes Screening for Adolescents in Community Health Settings. Abstract and Poster at: Pediatric academic societies meeting; 2012, Apr 27-30. Boston: Massachusetts, United States; 2012.

16. Shah S, Kublaoui BM, Oden JD, White PC: Screening for type 2 diabetes in obese youth. Pediatrics 2009, 124:573-578.

17. Roman R, Zeitler P: Oral glucose tolerance testing in asymptomatic obese children: more questions than answers. J Clin Endocrinol Metab 2008, 932:4228-4230.

18. Kleber M, Lass N, Papcke S, Wabitsch M, Reinehr T: One-year follow-up of untreated obese white children and adolescents with impaired glucose 
tolerance: high conversion rate to normal glucose tolerance. Diabet Med 2010, 27:516-521.

19. Moran A, Hardin D, Rodman D, Allen HF, Beall RJ, Borowitz D, et al:

Diagnosis, screening and management of cystic fibrosis related diabetes mellitus: a consensus conference report. Diabetes Res Clin Pract 1999, 45:61-73.

20. Brennan AL, Gyi KM, Wood DM, Hodson ME, Geddes DM, Baker EH: Relationship between glycosylated haemoglobin and mean plasma glucose concentration in cystic fibrosis. J Cyst Fibros 2006, 5:27-31.

21. Roberts WL, Safar-Pour S, De BK, Rohlfing CL, Weykamp CW, Little RR: Effects of Hemoglobin C and S traits on Glycohemoglobin measurements by Eleven Methods. Clin Chem 2005, 51:776-778.

22. Hanas R, John G: 2010 Consensus Statement on the Worldwide Standardization of the Hemoglobin A1c Measurement. Diabetes Care 2010, 33:1903-1904.

doi:10.1186/1687-9856-2012-31

Cite this article as: Kapadia and Zeitler: Hemoglobin A1c measurement for the diagnosis of Type 2 diabetes in children. International Journal of Pediatric Endocrinology 2012 2012:31.

\section{Submit your next manuscript to BioMed Central and take full advantage of:}

- Convenient online submission

- Thorough peer review

- No space constraints or color figure charges

- Immediate publication on acceptance

- Inclusion in PubMed, CAS, Scopus and Google Scholar

- Research which is freely available for redistribution 